\title{
Transcatheter mitral valve repair in proportionate and disproportionate functional mitral regurgitation - insights from a small cohort study
}

\author{
J. F. Ooms · M. L. Geleijnse - E. Spitzer · B. Ren · M. P. Van Wiechen · T. W. Hokken · J. Daemen • \\ P. P. T. de Jaegere · N. M. D. A. Van Mieghem
}

Accepted: 3 May 2021 / Published online: 8 June 2021

(C) The Author(s) 2021

\begin{abstract}
Background Functional mitral regurgitation (FMR) can be subclassified based on its proportionality relative to left ventricular function and end-diastolic volume. FMR proportionality could help identify responders to transcatheter edge-to-edge mitral valve repair (MitraClip) in terms of residual FMR and/or clinical improvement.

Methods This single-centre retrospective cohort study evaluated the feasibility of determining FMR proportionality in symptomatic heart failure patients with reduced left ventricular function who were treated with MitraClip for $\geq$ moderate-to-severe FMR. Baseline proportionate (pFMR) and disproportionate FMR (dFMR) were distinguished. Patient characteristics and MitraClip procedural outcomes were described. Results From an overall cohort of 81 eligible FMR patients, $23 / 81$ (28\%) had to be excluded due to missing transthoracic echocardiogram parameters, 22/81 were excluded based on FMR severity. The remaining cohort, of $36 / 81$ patients (44\%), could be classified into dFMR $(n=26)$ or pFMR $(n=10)$. Conduction disorders were numerically increased in dFMR. All cases requir-
\end{abstract}

\footnotetext{
Supplementary Information The online version of this article (https://doi.org/10.1007/s12471-021-01583-6) contains supplementary material, which is available to authorized users.

J. F. Ooms · M. P. Van Wiechen · T. W. Hokken · J. Daemen ·

P. P. T. de Jaegere - N. M. D. A. Van Mieghem ( $\varangle)$

Department of Interventional Cardiology, Thoraxcenter,

Erasmus University Medical Center, Rotterdam, The

Netherlands

n.vanmieghem@erasmusmc.nl
}

M. L. Geleijnse · E. Spitzer · B. Ren

Department of Echocardiography, Thoraxcenter, Erasmus

University Medical Center, Rotterdam, The Netherlands ing $>2$ clips were in the dFMR group and absence of FMR reduction occurred more frequently with dFMR. Point of view/Conclusion Important limitations in terms of imaging acquisition affect the translation of the FMR proportionality concept to a real-world data set. We did observe different demographic and FMR response patterns in patients with proportionate and disproportionate FMR that warrant further investigation.

Keywords Mitral valve $\cdot$ Functional mitral valve insufficiency $\cdot$ Valve repair

\section{Introduction}

As opposed to degenerative mitral regurgitation, treatment of functional mitral regurgitation (FMR) remains subject to debate [1]. FMR is commonly associated with heart failure and is mainly caused by geometrical or functional abnormalities of structures surrounding the intrinsically normal mitral valve [2]. Currently, FMR therapies-medical or otherwise-are directed at reducing or reversing the remodelling process of the left ventricle [3].

Two randomised controlled trials, COAPT [4] and MITRA-FR [5], investigated percutaneous mitral-valve repair with MitraClip (Abbot, Chicago, IL) in patients with systolic heart failure and $>$ moderate FMR. Conflicting results were reported in terms of mortality and heart failure related hospitalisation $[4,5]$. In COAPT, MitraClip treatment improved clinical outcome, while in MITRA-FR, no effect of MitraClip was found [6]. Differences in design and patient characteristics partially explain the discrepancies; in COAPT, patients presented with more severe FMR but less dilated left ventricles compared with patients in MITRA-FR.

Grayburn et al. devised a conceptual framework based on FMR proportionality and disproportional- 
ity to the dimensions and function of the left ventricle [7]. The hypothesis suggested that in a subgroup of FMR patients, the location and damage of affected left ventricular segments is unequally distributed. Consequently, segments involved in leaflet coaptation could be disproportionately affected, causing excessive FMR. Interventions targeting resynchronisation and/or mitral clipping might prove beneficial in the setting of disproportionate FMR (dFMR) [8]. The average COAPT FMR was deemed disproportionate to left ventricular dimensions and function as opposed to the average FMR in MITRA-FR that seemed proportionate (pFMR) [7].

This retrospective study aimed to evaluate the feasibility of the FMR proportionality concept in clinical practice as it has only been scarcely evaluated in a real-world MitraClip context [9, 10]. Furthermore, it describes patient demographics, procedural characteristics and immediate FMR reductions after MitraClip for patients with pFMR or dFMR.

\section{Methods}

We retrospectively determined FMR proportionality at baseline in all consecutive heart failure patients with reduced left ventricular ejection fraction (LVEF) who were treated with MitraClip for $\geq$ moderate-to-severe FMR at the Erasmus University Medical Center. Decision to treat was based on multidisciplinary heart team consensus.

Reasons for excluding patients from analysis were previous mitral valve repair or replacement, heart

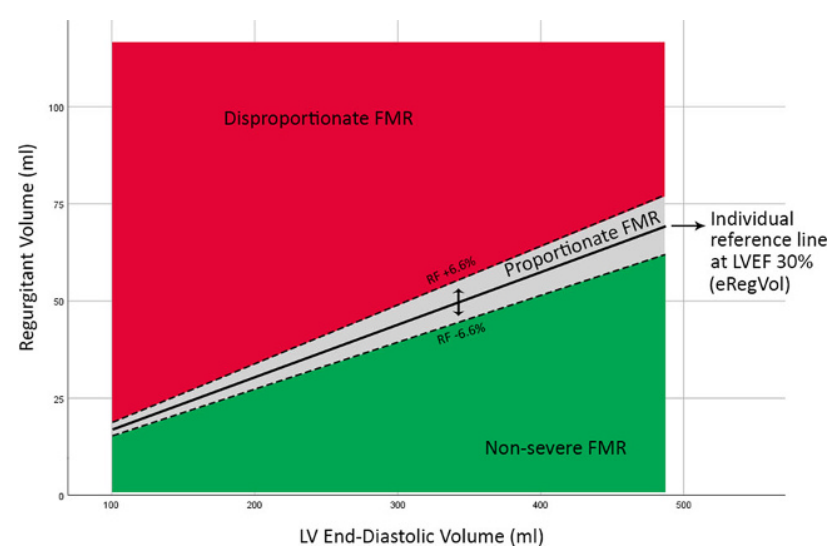

Fig. 1 Determining patient-specific proportionality of functional mitral regurgitation. Graph plotting the FMR regurgitant volume vs LVEDV. The black line shows the eRegVol for each given LVEDV at an LVEF of $30 \%$ and a regurgitant fraction of $50 \%$ (eRegVol=LVEDV $\times$ LVEF $\times 0.50$ ). A margin of $\pm 6.6 \%$ was instated (dotted lines). Any measured value of regurgitant volume within the red, grey or green zone are deemed disproportionate, proportionate or non-severe respectively. Of note, the reference line shifts for different, individual, values of LVEF. Details on used formulas are found in Appendix I in the Electronic Supplementary Material (ESM). FMR functional mitral regurgitation, LVEDV left ventricular end-diastolic volume, LVEF left ventricular ejection fraction, eRegVol expected regurgitant volume transplant or untreated significant coronary artery disease requiring revascularisation. Every patient signed informed consent for the clip procedure and use of related data in a dedicated database for study purposes. The study did not fall under the scope of the Medical Research Involving Human Subjects Act per Erasmus Medical Center Institutional Review Board.

The main study objective was to classify baseline FMR as proportionate or disproportionate. Patient and procedure characteristics were described per dFMR and pFMR cohort. Degree of post-procedural FMR reduction ( $\geq 1$ grade) and residual FMR at discharge were recorded.

\section{Echocardiographic assessment}

FMR aetiology and severity were evaluated by transthoracic echocardiogram (TTE) using an integrated approach of multiple qualitative and quantitative parameters including left atrial and ventricular size, jet features, pulmonary artery pressures, effective regurgitant orifice area (using the proximal isovelocity surface area [PISA]), and regurgitant volume (RegVol). RegVol was calculated from the effective regurgitant orifice area or alternatively the volumetric method, if the effective regurgitant orifice area was unavailable and in absence of aortic regurgitation [11, 12]. Reasons for the inability to measure effective regurgitant orifice area and regurgitant volumes were recorded.

\section{Assessment of functional mitral regurgitation proportionality}

Severe FMR was considered proportionate when the regurgitation was in line with expected RegVol based on left ventricular size and function derived from the Gorlin hydraulic orifice equation [7]. Using left ventricular end-diastolic volume, LVEF and a set regurgitation fraction of $50 \%$ we calculated the expected mitral regurgitation volume (eRegVol) of each individual patient. Similarly, we calculated the upper and lower limits of a grey zone around this value using regurgitation fraction $\pm 6.6 \%$ (Fig. 1, Appendix I in the Electronic Supplementary Material [ESM]). [9]. Subsequently, we compared the patient-specific eRegVol with the actual/measured RegVol. A measured RegVol above the eRegVol value was classified as disproportionate, a RegVol within the grey area (approximating the reference line) as proportionate and below as $<$ moderate-to-severe FMR (Fig. 1, Appendix I in the Electronic Supplementary Material [ESM]). Patients with < moderate-to-severe FMR were excluded from the analysis.

\section{Statistical methods}

Continuous variables are presented as mean \pm standard deviation for normally distributed data, or as median 
Fig. 2 Patient identification. Flow chart of the patient identification process showing that only a small percentage of patients in the prospective Mitraclip database was suitable for regurgitant orifice area, diastolic volume, LVEF left ventricular ejection fraction, RegVol regurgitant volume determination of proportionality. EROA effective LVEDV left ventricular end-
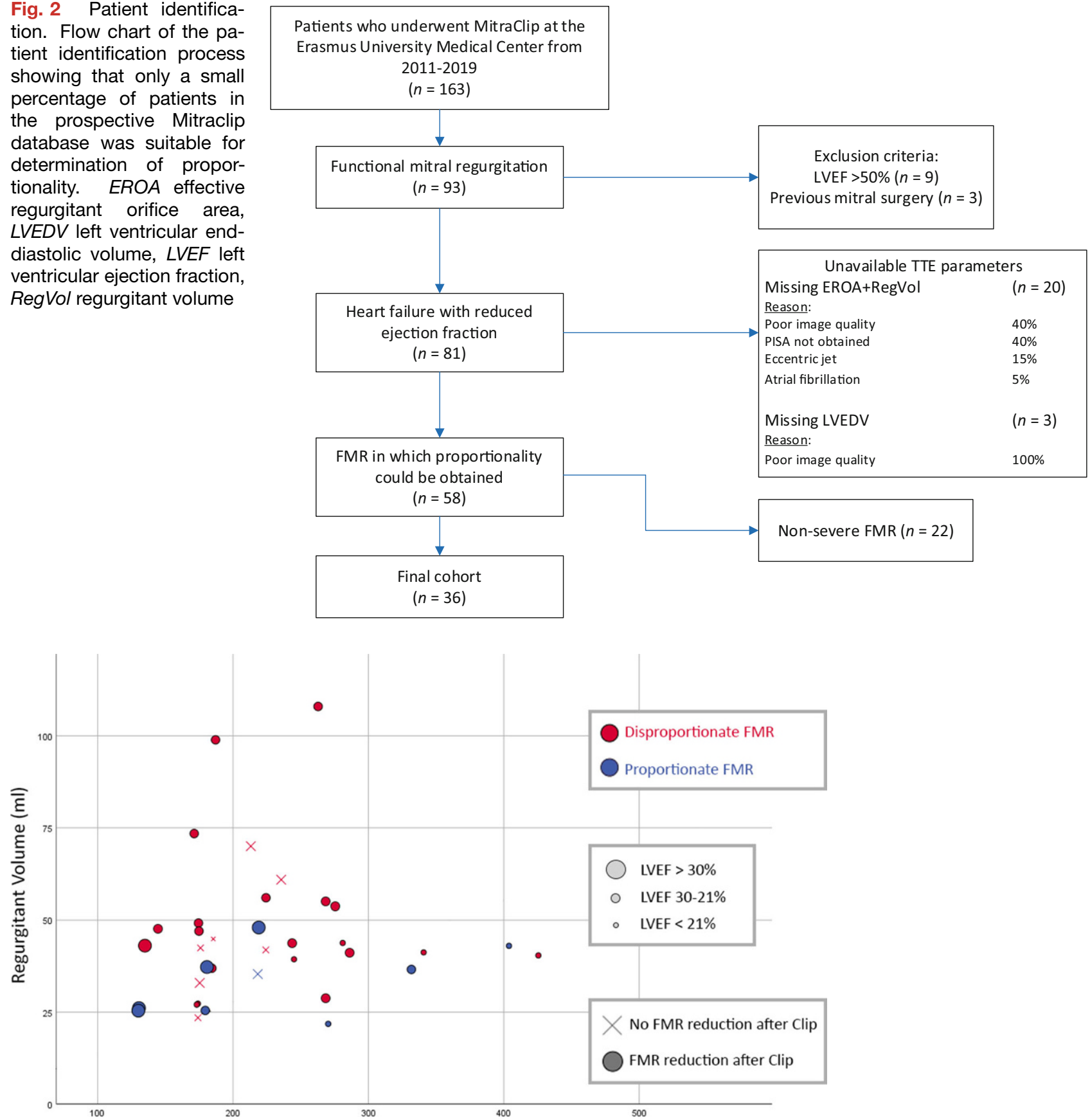

LV End-Diastolic Volume (ml)

Fig. 3 Functional mitral regurgitation severity and proportionality. Scatter plot depicting individual values of FMR regurgitant volume vs LVEDV at baseline. FMR proportionality was determined for 3 different groups of LVEF based on LVEF median and interquartile range (bubble size large, medium and small size depict an LVEF of $>30 \%, 21-30 \%$ and $<21 \%$ re-

with interquartile range [IQR] if non-normal. Timeto-event data of one-year mortality and heart failure related hospitalisation is reported using the KaplanMeier method. Statistical analyses were performed with SPSS 25.0 (IBM, Armonk, New York). spectively). Each individual FMR was classified as either disproportionate (red) or proportionate (b/ue). Patients with FMR reduction are depicted as a dot and patients without reduction are displayed as a cross. Dot/cross size is coded according to LVEF. FMR functional mitral regurgitation, LVEDV left ventricular end-diastolic volume, LVEF left ventricular ejection fraction

\section{Results}

We evaluated all patients who received MitraClip in our centre between 2011 and 2019 (Fig. 2). Ninetythree patients were diagnosed with FMR of which 12 were excluded based on exclusion criteria. FMR proportionality could not be determined in $23 / 81$ pa- 
Table 1 Baseline clinical characteristics

\begin{tabular}{|c|c|c|}
\hline & dFMR (26) & pFMR (10) \\
\hline \multicolumn{3}{|l|}{ Clinical } \\
\hline Age-yr & $71.8 \pm 10.5$ & $66.2 \pm 7.6$ \\
\hline Male & $17(65.4)$ & $8(80.0)$ \\
\hline Hypertension & $18(69.2)$ & $7(70.0)$ \\
\hline Diabetes mellitus & $8(30.8)$ & $5(50.0)$ \\
\hline Stroke/TIA & $1(3.8)$ & - \\
\hline Peripheral vascular disease & $7(26.9)$ & $2(20.0)$ \\
\hline COPD & $3(11.5)$ & $2(20.0)$ \\
\hline Creatinine clearance- $-\mathrm{ml} / \mathrm{min}$ & $38.2 \pm 16.3$ & $45.8 \pm 11.3$ \\
\hline Previous percutaneous coronary intervention & $14(53.8)$ & $6(60.0)$ \\
\hline Previous coronary artery bypass grafting & $10(38.5)$ & $3(30.0)$ \\
\hline Ischaemic cardiomyopathy & $20(76.9)$ & $8(80.0)$ \\
\hline Atrial fibrillation & $14(56.0)$ & $5(50.0)$ \\
\hline Cardiac resynchronisation therapy & $7(26.9)$ & $2(20.0)$ \\
\hline Implantable cardioverter defibrillator & $10(38.5)$ & $5(50.0)$ \\
\hline \multicolumn{3}{|l|}{ NYHA functional class } \\
\hline 1 & - & - \\
\hline$\|$ & $3(11.5)$ & $1(10.0)$ \\
\hline III & $14(53.9)$ & $7(70.0)$ \\
\hline IV & $9(34.6)$ & $2(20.0)$ \\
\hline STS-PROM score, median [IQR] & $4[2-10]$ & $2[1-5]$ \\
\hline \multicolumn{3}{|l|}{ Heart failure medication } \\
\hline Renin-angiotensin system antagonist & $14(53.8)$ & $6(60.0)$ \\
\hline Beta-blocker & $19(73.1)$ & $10(100.0)$ \\
\hline Mineralocorticoid antagonist & $19(73.1)$ & $8(80.0)$ \\
\hline Any diuretic & $26(100.0)$ & $10(100.0)$ \\
\hline Any inotropes ${ }^{\mathrm{a}}$ & - & - \\
\hline \multicolumn{3}{|c|}{$\begin{array}{l}\text { Data is presented in numbers with (\%) unless described otherwise. Plus- } \\
\text { minus values are means } \pm \text { SD } \\
\text { COPD chronic obstructive pulmonary disease, dFMR disproportionate func- } \\
\text { tional mitral regurgitation, NYHA New York Heart Association, } p F M R \text { pro- } \\
\text { portionate functional mitral regurgitation, TAVI transcatheter aortic valve } \\
\text { replacement, } T I A \text { transient ischaemic attack, STS-PROM Society of Thoracic } \\
\text { Surgeons predicted risk of mortality } \\
\text { aintravenously administered }\end{array}$} \\
\hline
\end{tabular}

tients (28\%) because effective regurgitant orifice area, RegVol or left ventricular end-diastolic volume could not be measured based on baseline TTE. In 22/81 patients (27\%), FMR was < moderate-to-severe (quantitatively obtained). The final cohort consisted of 36 patients, 26 with dFMR and 10 with pFMR (Fig. 3). Of note, RegVol was derived from the effective regurgitant orifice area in $51(88 \%)$ patients and from the volumetric method in $7(12 \%)$ patients. In the first half of the study period (i.e. $\leq 2015), 16$ (44\%) patients were included, 20 (56\%) patients were included in the second half.

Baseline patient demographics, electrocardiographic and echocardiographic data are reported in Tab. 1 and 2. Procedural results are reported in Tab. 3 . Several numerical differences were observed between dFMR and pFMR groups. Older age, kidney dysfunction, higher Society of Thoracic Surgeons (STS) score and prolonged QRS duration was more frequent in
Table 2 Baseline ventricular conduction and echocardiographic parameters

\begin{tabular}{|c|c|c|}
\hline & dFMR (26) & pFMR (10) \\
\hline \multicolumn{3}{|l|}{ Baseline ECG variable } \\
\hline QRS duration-ms & 150 [121-191] & $128[112-186]$ \\
\hline QRS duration in non-CRT group-ms & 134 [117-182] & $123[111-136]$ \\
\hline CRT or $\mathrm{QRS} \geq 150 \mathrm{~ms}$ & $13(50.0)$ & $3(30.0)$ \\
\hline Left bundle branch block & $16(61.5)$ & $6(60.0)$ \\
\hline \multicolumn{3}{|l|}{ Baseline TTE variable } \\
\hline LVEF-\% & $24.3 \pm 1.3$ & $30.2 \pm 9.3$ \\
\hline LVESV—ml & 150 [129-206] & 142 [95-227] \\
\hline LVEDV—ml & $200[175-264]$ & $219[167-286]$ \\
\hline LA volume index-ml/ $\mathrm{m}^{2}$ & $76.7 \pm 17.7$ & $61.8 \pm 14.5$ \\
\hline \multicolumn{3}{|l|}{ MR grade—no. (\%) } \\
\hline - Moderate-to-severe & 7 (26.9) & $3(30.0)$ \\
\hline - Severe & $19(73.1)$ & $7(70.0)$ \\
\hline EROA-mm ${ }^{2}$ & 34.1 [29-43] & 22.5 [20-27] \\
\hline MR VTI—cm & $138.9 \pm 27.8$ & $148.8 \pm 11.0$ \\
\hline Mitral RegVol—ml & $43[40-55]$ & 32 [25-39] \\
\hline
\end{tabular}

Plus-minus values are means $\pm \mathrm{SD}$. Other values are median with (interquartile range) or numbers with (\%) unless explicitly stated otherwise $C R T$ cardiac resynchronisation therapy, $d F M R$ disproportionate functional mitral regurgitation, ECG electrocardiogram, EROA effective regurgitant orifice area, $L A$ left atrium, LVEDD left ventricular end-diastolic diameter, LVEDV left ventricular end-diastolic volume; $L V E F$ left ventricular ejection fraction; LVESD left ventricular end-systolic diameter, LVESV left ventricular end-systolic volume, $M R$ mitral regurgitation, $p F M R$ proportionate functional mitral regurgitation, RegVol regurgitant volume, SPAP systolic pulmonary artery pressure, $T R$ tricuspid regurgitation, $T T E$ transthoracic echocardiography, VT/velocity time integral

dFMR. In dFMR, LVEF and left ventricular end-diastolic volume appeared lower, while total number of clips per procedure and residual FMR severity at discharge were higher (Fig. 3).

Median follow-up was 464 days (IQR 181-935). One year mortality was $26.5 \%(n=9)$. Heart failure related hospitalisation rate within 1 year after MitraClip was 25.7\% ( $n=8)$. During follow-up, New York Heart Association (NYHA) class improved in $69 \%$ of patients (see Tab. 1 in the Electronic Supplementary Material [ESM]).

\section{Discussion}

Our study applied the FMR proportionality concept to heart failure patients who underwent MitraClip therapy in everyday practice and demonstrated that the quality and completeness of echocardiography studies were often suboptimal and lacked essential recordings to determine FMR proportionality. We could categorise only $44 \%$ of the overall study cohort into pFMR/dFMR.

Several issues affected the overall sample size of the study. First, methods to determine FMR proportionality rely on effective regurgitant orifice area and RegVol for quantification $[9,10]$, which may not be straightforward to obtain in FMR [13-15]. In this real-world sample, effective regurgitant orifice area 
Table 3 Procedural characteristics

\begin{tabular}{|c|c|c|}
\hline Procedural variable & dFMR (26) & pFMR (10) \\
\hline \multicolumn{3}{|l|}{ No. clips } \\
\hline 1 & $9(34.6)$ & $5(50.0)$ \\
\hline 2 & $13(50.0)$ & $5(50.0)$ \\
\hline 3 & $3(11.5)$ & - \\
\hline 4 & $1(3.8)$ & - \\
\hline Technical success ${ }^{\mathrm{a}}$ & $23(88.5)$ & $10(100.0)$ \\
\hline \multicolumn{3}{|l|}{ Mitral regurgitation at discharge $\mathrm{e}^{\mathrm{b}}$} \\
\hline Trace & $1(3.8)$ & - \\
\hline Mild & $12(46.1)$ & $4(40.0)$ \\
\hline Mild-to-moderate & $2(7.7)$ & $3(30.0)$ \\
\hline Moderate & $5(19.2)$ & $1(10.0)$ \\
\hline Moderate-to-severe & $1(3.8)$ & $1(10.0)$ \\
\hline Severe & $5(19.2)$ & $1(10.0)$ \\
\hline Discharge MR $\geq$ moderate $^{\mathrm{b}}$ & $11(44.0)$ & $3(33.3)$ \\
\hline \multicolumn{3}{|l|}{ Change in MR discharge versus baseline } \\
\hline None & $5(19.2)$ & $1(10.0)$ \\
\hline 1 Grade & $3(11.5)$ & $2(20.0)$ \\
\hline$\geq 2$ Grades & $18(69.2)$ & $7(70.0)$ \\
\hline Mitral mean gradient at discharge $\mathrm{e}^{\mathrm{b}}-\mathrm{mm} \mathrm{Hg}$ & $3.7 \pm 1.7$ & $3.2 \pm 1.3$ \\
\hline \multicolumn{3}{|c|}{$\begin{array}{l}\text { Data is presented in numbers with (\%) unless described otherwise. Plus- } \\
\text { minus values are means } \pm \text { SD } \\
d F M R \text { disproportionate functional mitral regurgitation, MR mitral regurgita- } \\
\text { tion, } p F M R \text { proportionate functional mitral regurgitation, MVARC Mitral Valve } \\
\text { Academic Research Consortium criteria } \\
{ }^{a} \text { Defined according to MVARC criteria } \\
{ }^{b} \text { Obtained with transthoracic echocardiography. }\end{array}$} \\
\hline
\end{tabular}

and RegVol appeared missing in a relevant number of cases. Low image quality and absence of essential images required to perform the measurements were the main reasons for missing data. Other methods to derive RegVol were often unavailable or deemed unreliable (i.e. coinciding aortic regurgitation). The minimum amount of image recordings required for proportionality assessment is five (for effective regurgitant orifice area, VTI, left ventricular end-diastolic volume [biplane] and LVEF). Although the requirement of these images and subsequent measurements are established in society guidelines [12, 16], these measurements appeared challenging and often overlooked.

Second, $27 \%$ of our initial cohort were excluded because of $<$ moderate-to-severe FMR. This may suggest that the multi-disciplinary heart team may have considered MitraClip as a last resort therapy in the context of end-stage heart failure with < moderate-to-severe FMR. The application of MitraClip for less severe FMR in heart failure is attractive but not the scope of this study.

The ratio between effective regurgitant orifice area and left ventricular end-diastolic volume may be an alternative, simplified method to identify dFMR, yet lacks established cut-off values to separate dFMR from pFMR and disregards LVEF that seemed essential to interpret FMR in the original framework [10]. We, therefore, opted to use patient-specific reference lines derived from each individual's RegVol, left ventricular end-diastolic volume and LVEF to determine proportionality. Further validation of this FMR proportionality concept requires larger samples and optimised echocardiography protocols to assure complete mitral regurgitation analysis. Clearly, this framework relies on generally adopted, standardised, echocardiographic acquisition protocols including all required images for quantification.

Although applying the proportionality framework to real-world data proved challenging, some interesting observations were made: 1) dFMR was characterised by conduction disorders and more severe left ventricular dysfunction, 2) dFMR may require more clips, and 3) absence of FMR reduction occurred more frequently with dFMR.

Our data seem to confirm that focal left ventricular remodelling and/or dyssynchrony are fundamental mechanisms that underlie dFMR development. There was a higher prevalence of either cardiac resynchronisation therapy implantation or QRS $\geq 150$ ms with dFMR. Prolonged QRS duration marks ventricular dyssynchrony [17, 18], while cardiac resynchronisation therapy has been demonstrated to be less efficacious in ischaemic cardiomyopathy [19]. In our dFMR cohort, $>75 \%$ had ischaemic heart failure, which might explain persistent cardiac dyssynchrony, non-response to cardiac resynchronisation therapy, and dFMR.

All cases with $>2$ clips were in the dFMR group and in spite of using more clips, dFMR reduction appeared less. This finding may result from more extensive mitral regurgitation at baseline. However, clipping does not correct cardiac dyssynchrony, which is associated with residual mitral regurgitation after surgical repair [20].

The retrospective nature of our analysis holds inherent selection bias. To determine FMR proportionality, multiple quantitative echocardiographic parameters are required, each associated with measurement inaccuracies. Importantly, FMR severity in clinical practice is graded using a multi-parametric approach including quantitative and qualitative parameters and is often based on multiple echocardiography studies that may reveal dynamic mitral regurgitation. Standardised comprehensive TTE acquisition protocols and experienced imaging specialists seem essential. This study sample was not powered to make any statistical comparison and should be considered as descriptive and hypothesis generating. We believe our study adds perspective to the application of the concept of mitral regurgitation proportionality in clinical practice and warrants further study. A multicentre initiative is ongoing to complement these single-centre observations. 


\section{Conclusion}

Important limitations in terms of imaging acquisition affected the translation of the FMR proportionality concept to a real-world data set. We did observe different demographic and FMR response patterns in patients with proportionate and disproportionate FMR that warrants further investigation.

Conflict of interest N.M.D.A. Van Mieghem has received a research grant support from Boston Scientific, Edwards Lifesciences, Abbot Vascular and PulseCath B.V. which were received for activities outside the submitted work. J. Daemen received institutional grant/research support from AstraZeneca, Abbott Vascular, Boston Scientific, ACIST Medical, Medtronic, Pie Medical, ReCor medical and PulseCath, and consultancy and speaker fees from ACIST medical, Boston Scientific, ReCor Medical, Pie Medical, Medtronic and Pulse Cath. J.F. Ooms, M.L. Geleijnse, E. Spitzer, B. Ren, M.P. Van Wiechen, T.W. Hokken and P.P.T. de Jaegere declare that they have no competing interests.

Open Access This article is licensed under a Creative Commons Attribution 4.0 International License, which permits use, sharing, adaptation, distribution and reproduction in any medium or format, as long as you give appropriate credit to the original author(s) and the source, provide a link to the Creative Commons licence, and indicate if changes were made. The images or other third party material in this article are included in the article's Creative Commons licence, unless indicated otherwise in a credit line to the material. If material is not included in the article's Creative Commons licence and your intended use is not permitted by statutory regulation or exceeds the permitted use, you will need to obtain permission directly from the copyright holder. To view a copy of this licence, visit http://creativecommons.org/licenses/by/4.0/.

\section{References}

1. Baumgartner H, Falk V, Bax JJ, et al. 2017 ESC/EACTS Guidelines for the management of valvular heart disease. Eur Heart J. 2017;38:2739-91.

2. Levine RA, Schwammenthal E. Ischemic mitral regurgitation on the threshold of a solution: from paradoxes to unifying concepts. Circulation. 2005;112:745-58.

3. Ponikowski P, Voors AA, Anker SD, et al. 2016ESCGuidelines for the diagnosis and treatment of acute and chronic heart failure: The Task Force for the diagnosis and treatment of acute and chronic heart failure of the European Society of Cardiology (ESC) Developed with the special contribution of the Heart Failure Association (HFA) of the ESC. Eur HeartJ. 2016;37:2129-200.

4. Stone GW, Lindenfeld J, Abraham WT, et al. Transcatheter mitral-valve repair in patients with heart failure. N Engl J Med. 2018;379:2307-18

5. Obadia JF, Messika-Zeitoun D, Leurent G, et al. Percutaneous repair or medical treatment for secondary mitral regurgitation. NEngl J Med. 2018;379:2297-306.
6. Iung B, Armoiry X, Vahanian A, et al. Percutaneous repair or medical treatment for secondary mitral regurgitation: outcomes at 2 years. Eur J Heart Fail. 2019;21:1619-27.

7. Grayburn PA, Sannino A, Packer M. Proportionate and disproportionate functional mitral regurgitation: a new conceptual framework that reconciles the results of the MITRA-FR and COAPT trials. JACC Cardiovasc Imaging. 2019;12:353-62.

8. Packer M, Grayburn PA. Contrasting effects of pharmacological, procedural, and surgical interventions on proportionate and disproportionate functional mitral regurgitation in chronic heartfailure. Circulation. 2019;140:779-89.

9. Bartko PE, Heitzinger G, Arfsten H, et al. Disproportionate functional mitral regurgitation: advancing a conceptual framework to clinical practice. JACC Cardiovasc Imaging. 2019;12:2088-90.

10. Adamo M, Cani DS, Gavazzoni M, et al. Impact of disproportionate secondary mitral regurgitation in patients undergoing edge-to-edge percutaneous mitral valve repair. EuroIntervention. 2020; https://doi.org/10.4244/EIJ-D19-01114.

11. Lancellotti P, Moura L, Pierard LA, et al. European Association of Echocardiography recommendations for the assessment of valvular regurgitation. Part 2: mitral and tricuspid regurgitation (native valve disease). Eur J Echocardiogr. 2010;11:307-32.

12. Lancellotti P, Tribouilloy C, Hagendorff A, et al. Recommendations for the echocardiographic assessment of native valvular regurgitation: an executive summary from the European Association of Cardiovascular Imaging. Eur Heart J Cardiovasc Imaging. 2013;14:611-44.

13. Beigel R, Siegel RJ. Should the guidelines for the assessment of the severity of functional mitral regurgitation be redefined? JACC Cardiovasc Imaging. 2014;7:313-4.

14. Grayburn PA, Carabello B, Hung J, et al. Defining "severe" secondary mitral regurgitation: emphasizing an integrated approach. J Am Coll Cardiol. 2014;64:2792-801.

15. Ray S. The echocardiographic assessment of functional mitral regurgitation. Eur J Echocardiogr. 2010;11:i11-7.

16. Zoghbi WA, Adams D, Bonow RO, et al. Recommendations for noninvasive evaluation of native valvular regurgitation: a report from the American Society of Echocardiography developed in collaboration with the Society for Cardiovascular Magnetic Resonance. J Am Soc Echocardiogr. 2017;30:303-71.

17. Grines CL, Bashore TM, Boudoulas H, et al. Functional abnormalities in isolated left bundle branch block. The effect of interventricular asynchrony. Circulation. 1989;79:845-53.

18. Vancheri F, Vancheri S, Henein M. Relationship between QRS measurements and left ventricular morphology and function in asymptomatic individuals. Echocardiography. 2018;35:301-7.

19. Bleeker GB, Kaandorp TA, Lamb HJ, et al. Effect of posterolateral scar tissue on clinical and echocardiographic improvement after cardiac resynchronization therapy. Circulation. 2006;113:969-76.

20. Petrus AHJ, Dekkers OM, Tops LF, et al. Impact of recurrent mitral regurgitation after mitral valve repair for functional mitral regurgitation: long-term analysis of competing outcomes. Eur HeartJ. 2019;40:2206-14. 